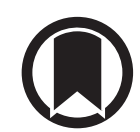

CrossMark

\title{
Neutrophils disturb pulmonary microcirculation in sepsis-induced acute lung injury
}

\author{
Inwon Park (1) 1,5, Mingyo Kim²,2, Kibaek Choe ${ }^{3,4}$, Eunjoo Song ${ }^{3,4}$, Howon Seo ${ }^{3,4}$, \\ Yoonha Hwang ${ }^{3,4}$, Jinhyo Ahn ${ }^{3,4}$, Seung-Hyo Lee ${ }^{1}$, Jae Hyuk Lee ${ }^{5}$, You Hwan Jo ${ }^{5}$, \\ Kyuseok Kim ${ }^{5}$, Gou Young Koh ${ }^{1,6,7}$ and Pilhan Kim ${ }^{1,3,4,7}$
}

Affiliations: ${ }^{1}$ Graduate School of Medical Science and Engineering, Korea Advanced Institute of Science and Technology (KAIST), Daejeon, Republic of Korea. ${ }^{2}$ Division of Rheumatology, Dept of Internal Medicine, Gyeongsang National University School of Medicine, Jinju, Republic of Korea. ${ }^{3}$ Graduate School of Nanoscience and Technology, Korea Advanced Institute of Science and Technology (KAIST), Daejeon, Republic of Korea. ${ }^{4} \mathrm{KI}$ for Health Science and Technology (KIHST), Korea Advanced Institute of Science and Technology (KAIST), Daejeon, Republic of Korea. ${ }^{5}$ Dept of Emergency Medicine, Seoul National University Bundang Hospital (SNUBH), Seongnam-si, Republic of Korea. ${ }^{6}$ Center for Vascular Research, Institute for Basic Science (IBS), Daejeon, Republic of Korea. ${ }^{7}$ Joint lead authors.

Correspondence: Pilhan Kim, Graduate School of Nanoscience and Technology, KAIST, 291 Daehak-ro, Daejeon, 34141, Republic of Korea. E-mail: pilhan.kimakaist.ac.kr

@ERSpublications

Neutrophils induce dead space in the pulmonary microcirculation in sepsis-induced ALI, recovered by a Mac-1 inhibitor http://ow.ly/vUzO30nbUyU

Cite this article as: Park I, Kim M, Choe K, et al. Neutrophils disturb pulmonary microcirculation in sepsis-induced acute lung injury. Eur Respir J 2019; 53: 1800786 [https://doi.org/10.1183/13993003.007862018].

ABSTRACT The lung is highly vulnerable during sepsis, yet its functional deterioration accompanied by disturbances in the pulmonary microcirculation is poorly understood. This study aimed to investigate how the pulmonary microcirculation is distorted in sepsis-induced acute lung injury (ALI) and reveal the underlying cellular pathophysiologic mechanism.

Using a custom-made intravital lung microscopic imaging system in a murine model of sepsis-induced ALI, we achieved direct real-time visualisation of the pulmonary microcirculation and circulating cells in vivo. We derived the functional capillary ratio (FCR) as a quantitative parameter for assessing the fraction of functional microvasculature in the pulmonary microcirculation and dead space.

We identified that the FCR rapidly decreases in the early stage of sepsis-induced ALI. The intravital imaging revealed that this decrease resulted from the generation of dead space, which was induced by prolonged neutrophil entrapment within the capillaries. We further showed that the neutrophils had an extended sequestration time and an arrest-like dynamic behaviour, both of which triggered neutrophil aggregates inside the capillaries and arterioles. Finally, we found that Mac-1 (CD11b/CD18) was upregulated in the sequestered neutrophils and that a Mac-1 inhibitor restored the FCR and improved hypoxaemia.

Using the intravital lung imaging system, we observed that Mac-1-upregulated neutrophil aggregates led to the generation of dead space in the pulmonary microcirculation that was recovered by a Mac-1 inhibitor in sepsis-induced ALI.

This article has supplementary material available from erj.ersjournals.com

Received: April 262018 | Accepted after revision: Dec 272018

Copyright $\odot$ ERS 2019. This version is distributed under the terms of the Creative Commons Attribution Non-Commercial Licence 4.0. 


\section{Introduction}

Sepsis is one of the most unaffordable conditions of hospitalisation and the foremost contributor to hospital death, representing a major worldwide health burden [1,2]. Sepsis is a syndrome characterised by a dysregulated response of the host to invading pathogens that involves haemodynamic alterations which lead to multiple life-threatening organ dysfunctions $[3,4]$. Among the injured organs, the lung is the first and most frequent organ to fail. Accompanying acute respiratory distress syndrome (ARDS), which is the clinical term for acute lung injury (ALI), is one of the most critical prognostic factors for mortality in patients with sepsis [5]. Despite intense research efforts aimed at treating sepsis-induced ARDS, a lung supportive ventilation strategy remains a mainstay of treatment, and no effective therapies aimed at the microcirculation to directly improve the ventilation-perfusion mismatch are available in ARDS [6]. Although "dead space" assessment could provide significant clinical value in ARDS [6, 7], to date it has remained a hypothesised impairment in lung alveoli that are ventilated but not perfused. Admittedly, ARDS is a poorly understood syndrome with regard to the association between lung injury and microcirculation $[6,8]$. Recently, a study reported evidence of thrombi in the pulmonary vasculature but the findings were limited because it was an ex vivo study; the in vivo process of neutrophil influx and the consequent disturbance in the pulmonary microcirculation remain to be investigated $[9,10]$.

Unregulated recruitment and activation of neutrophils could induce organ injury through the release of inflammatory mediators, including cytokines and reactive oxygen species (ROS) [11, 12]. Yet, our knowledge on the detailed dynamic behaviour of neutrophils in the pulmonary microcirculation is mostly limited to speculation gleaned from observations in the systemic circulation [13]. Because the diameter of neutrophils is greater than that of the pulmonary capillaries, neutrophils must deform to pass through the capillaries, which is a relatively time-consuming process [14]. This process, referred to as neutrophil sequestration, was originally described for cells other than the freely circulating group of neutrophils within the lung and has been observed, to some extent, using macroscopic radiolabelling imaging devices [15]. Previous studies have demonstrated neutrophil sequestration in lung capillaries; however, the mechanism by which a neutrophil sequestration event leads to ARDS remains unknown [16, 17]. Given the importance and obscurity of the pulmonary microcirculation in ARDS, it is imperative that we understand the changes occurring in the pulmonary microcirculation, including the dynamic behaviour of neutrophils, to elucidate the pathophysiology; this may lead to novel treatment strategies for sepsis-induced ARDS [18].

To investigate the pulmonary microcirculation in sepsis-induced ARDS, we used a custom-made video-rate laser scanning confocal microscope in combination with a microsuction-based pulmonary imaging window [19, 20]. Using the intravital lung imaging system, we directly identified an alteration in microcirculatory perfusion in a murine sepsis-induced ALI model and demonstrated the role of Mac-1-upregulated neutrophils in the pulmonary microcirculation, which suggests the clinical potential of a Mac-1 inhibitor as a therapeutic drug for sepsis-induced ALI.

\section{Materials and methods}

Mice

All animal experiments were performed in accordance with standard guidelines for the care and use of laboratory animals and were approved by the Institutional Animal Care and Use Committee (IACUC) of Korea Advanced Institute of Science and Technology (protocol no. KA2014-30 and KA2016-55). All mice were individually housed in ventilated, temperature- and humidity-controlled cages $\left(22.5^{\circ} \mathrm{C}, 52.5 \%\right)$ under a 12:12 h light:dark cycle and provided with standard diet and water ad libitum. For experimental use, 8-20-week-old male mice $(20-30 \mathrm{~g})$ were used in this study. C57BL/6N mice were purchased from OrientBio (Suwon, Republic of Korea). Tie2-GFP mice, in which the green fluorescent protein (GFP) is expressed under an endothelium-specific Tie2 promoter, were purchased from Jackson Laboratory (stock no. 003658; Jackson Laboratory, Bar Harbor, ME, USA). LysM ${ }^{\mathrm{GFP} /+}$ mice were generously provided by Professor M. Kim (University of Rochester, Rochester, NY, USA).

\section{Animal models}

To generate a sepsis-induced ALI model [12], a high-dose lipopolysaccharide (LPS) (10 mg.kg ${ }^{-1}$, Escherichia coli serotype 055:B5, L2880; Sigma-Aldrich, St Louis, MO, USA) or a high-grade caecal ligation and puncture (CLP) model was utilised. Details are provided in the supplementary material.

\section{Fluorescent dye and antibody utilised in intravital imaging}

In vivo labelling of erythrocytes, vasculature, neutrophils (Ly6G), CD11b, CD18, dihydroethidium (DHE) and modelling of neutrophil depletion and Mac-1 inhibition treatment are described in the supplementary material. 
Flow cytometry, histological analysis and arterial blood gas analysis

Details are provided in the supplementary material.

Imaging system and intravital pulmonary imaging

To visualise in vivo pulmonary microcirculation through a pulmonary imaging window, a custom-made video-rate laser scanning confocal microscopy system was implemented [21, 22]. Additional details of the imaging system and preparation of the mouse for intravital pulmonary imaging are provided in the supplementary material.

\section{Image processing}

Functional capillary imaging analysis was performed using a real-time video of DiD-labelled erythrocytes flowing in capillaries. After splitting the colours in the video, sequential images of channels detecting DiD were processed by a median filter with a radius of two pixels to enhance the signal-to-noise ratio. A maximal intensity projection of 600-900 frames (20-30 s) was generated to show the functional capillary perfused by erythrocytes. The functional capillary ratio (FCR) was determined by calculating the ratio of the functional capillary area (DiD-labelled red blood cells) to the total capillary area (vessel area detected by Tie2 or Dextran signalling). All image processing to calculate FCR was performed by ImageJ (https://imagej.nih.gov/ij/). Image rendering with three-dimensional reconstruction, track analysis of erythrocytes and neutrophils, and plotting track displacement was conducted using IMARIS 8.2 (Bitplane, Zurich, Switzerland). Additional details of image processing are provided in the supplementary material.

\section{Statistical analysis}

All data are presented as mean $\pm \mathrm{SD}$ or median (interquartile range), as appropriate to represent the values of the group. Statistical differences between means or medians were determined by unpaired two-tailed t-tests, Mann-Whitney tests, one-way ANOVAs with post hoc Holm-Sidak's multiple comparisons or Kruskal-Wallis tests with post hoc Dunn's multiple comparison tests, as appropriate. Statistical significance was set at $\mathrm{p}<0.05$, and analyses were performed with Prism 6.0 (GraphPad, San Diego, CA, USA).

\section{Results}

\section{The FCR is decreased in the pulmonary microcirculation in sepsis-induced ALI}

The cellular dynamics in the pulmonary microcirculation were investigated in vivo in mice using a custom-made intravital video-rate laser scanning confocal microscopy system $[19,20]$. Erythrocytes were sampled from a Tie2-GFP transgenic donor mouse by cardiac puncture. After labelling with DiD, adoptive transfer was performed into a syngenic recipient mouse by the tail vein (figure 1a) [23]. Rapidly flowing DiD-labelled erythrocytes were clearly visible in real-time inside the pulmonary vessel in which the endothelial cells were labelled with GFP, enabling the acquisition of spatiotemporal information on the flow trajectory and velocity of individual erythrocytes (figure 1b). The track information was acquired in vivo for multiple erythrocytes simultaneously and analysed (figure 1c and supplementary video S1).

Next, we used a murine endotoxin-induced indirect lung injury model to investigate changes in the erythrocyte flow in the pulmonary microcirculation in sepsis [12]. Intravital pulmonary imaging was performed $6 \mathrm{~h}$ after the administration of $10 \mathrm{mg} \cdot \mathrm{kg}^{-1}$ of intraperitoneal LPS, using DiD-labelled erythrocytes that were injected during the imaging. Though no significant difference was found in the mean velocity of the erythrocytes between the control and LPS groups (figure 1d), the erythrocyte perfusion pattern dramatically changed in the LPS-treated mice (supplementary video S2). To determine and quantify the perfusion area, erythrocytes in sequential images from 600 frames $(20 \mathrm{~s})$ were presented in a maximal intensity projection manner (supplementary figure S1a, b). The control group exhibited widespread and diffuse characteristics of perfusion, whereas the distribution of perfusion in the LPS-treated mice was more concentrated and overlapped with arterioles and a few capillaries (figure 1e).

We then defined the parameter FCR as a quantitative measure of the pulmonary microcirculation, which was calculated from the ratio of the functional capillary area, as determined by the erythrocyte trajectory (DiD, red), to the total capillary area (Tie2, green). A decreased FCR, which represents abnormal perfusion, was observed in the sepsis-induced ALI group even though there was not a large difference in the total capillary area (figure 1f, g). Furthermore, we confirmed that our FCR results correspond to hypoxaemia and hypercapnia from the arterial blood gas analysis in the left ventricle (figure 1h, i) [12].

\section{Entrapped neutrophils in pulmonary capillaries induce microcirculatory disturbances in sepsis-induced ALI}

During the imaging to measure the FCR in sepsis-induced ALI mice, we observed several sites of capillary obstruction in the magnified view. These obstructions were induced by objects inside the capillaries that could represent the primary pathophysiological mechanism underlying the decreased FCR in the early 


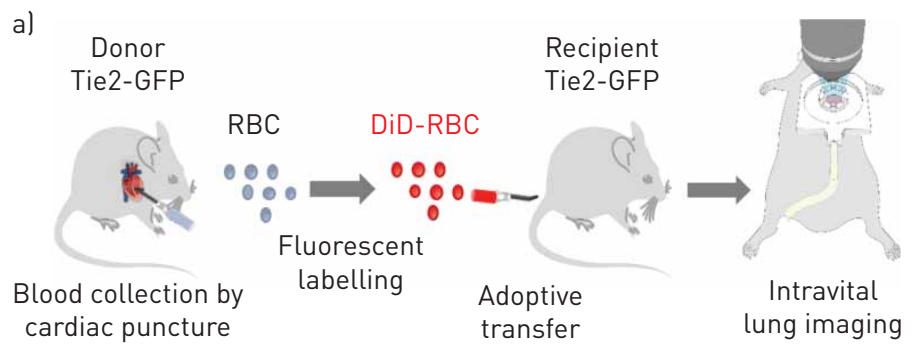

b)
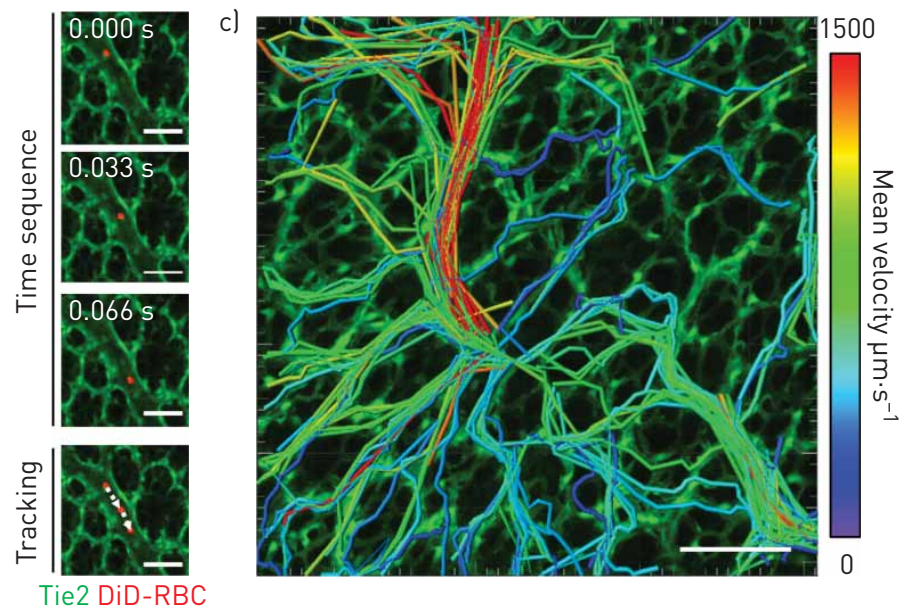

e)
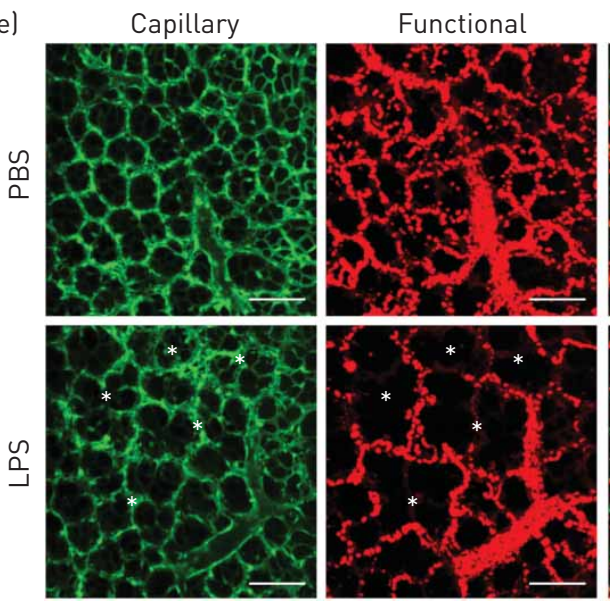

Tie2

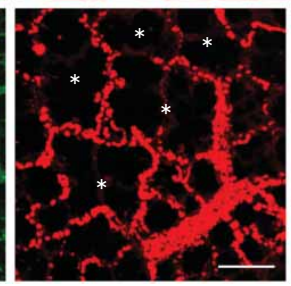

DiD-RBC

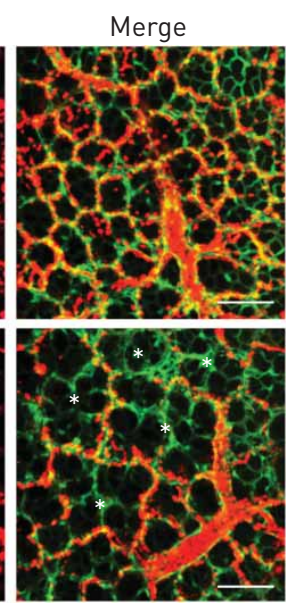

Tie2 DiD-RBC

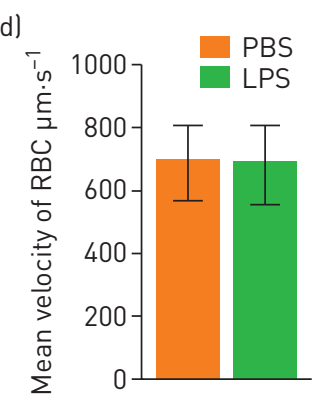

f)

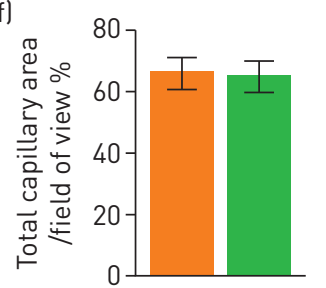

g)

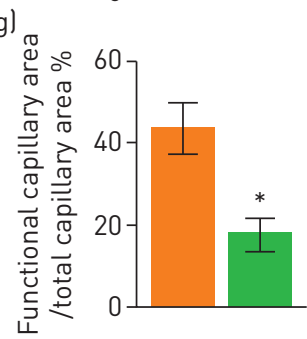

h)

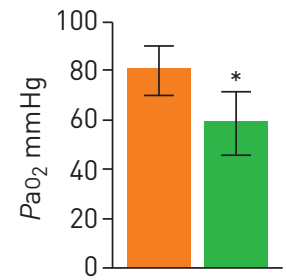

i)

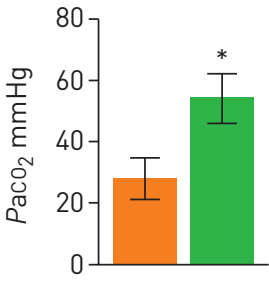

FIGURE 1 Impaired pulmonary microcirculation in sepsis-induced acute lung injury revealed by the functional capillary ratio. a) Schematics of intravital lung imaging experiment for pulmonary microcirculation visualisation with adoptive transfer of DiD-labelled erythrocytes. b) Sequential images of rapidly flowing DiD-labelled erythrocyte (red) inside the pulmonary vessel (green) in Tie2-GFP mouse obtained in video rate (30 frames $\cdot \mathrm{s}^{-1}$ ). Scale bars, $100 \mu \mathrm{m}$. Time elapsed is indicated. c) Velocity colour-coded track analysis of DiD-labelled erythrocyte in pulmonary microcirculation. Colour represents the mean velocity of each segment of erythrocyte track (supplementary video S1). Scale bar, $100 \mu \mathrm{m}$. d) Comparison of mean velocity of red blood cells (RBC) between the PBS and lipopolysaccharide (LPS) $\left(10 \mathrm{mg} \cdot \mathrm{kg}^{-1}\right)$ groups in the pulmonary microcirculation ( $n=30,10$ fields of view (FOV) per mouse, three mice per group, two-tailed t-test, $p=0.8157$ ). e) Representative functional capillary imaging in the PBS and LPS groups (supplementary video S2). The functional capillary was revealed by maximal intensity projection of real-time DiD-labelled erythrocyte imaging (supplementary figure S1a, b). White asterisks indicate dead spaces where the trajectory of the erythrocyte was not observed. Scale bars, $100 \mu \mathrm{m}$. $\mathrm{f}, \mathrm{g}$ ) Comparisons of the ratio of the total capillary area ( $f$ ) and the functional capillary area (g) between the PBS and LPS groups ( $n=30,10$ FOV per mouse, three mice per group, two-tailed t-test, $\left.{ }^{*} \mathrm{p}<0.05\right)$. h, i) Comparisons of the arterial oxygen $\left(\mathrm{PaO}_{2}\right)$ and carbon dioxide $\left(\mathrm{PaCO}_{2}\right)$ tension at $6 \mathrm{~h}$ after LPS between the PBS $(n=6)$ and LPS $(n=16)$ groups (Mann-Whitney test, $\left.{ }^{*} p<0.05\right)$. Data are presented as mean \pm SD. GFP: green fluorescent protein. 
stage of ALI development. Because neutrophils rapidly respond to systemic inflammation, we postulated that the objects that induced the obstruction could be neutrophils [13]. Real-time intravital imaging of the pulmonary capillaries in a naive $\mathrm{LysM}^{\mathrm{GFP} /+}$ animal showed transient entrapment of neutrophils in the capillary during circulation (figure $2 \mathrm{a}$ and supplementary video S3) $[24,25]$. During the period in which the capillaries became obstructed, circulating cells, which were assumed to be predominantly erythrocytes, could not flow through the capillaries. However, capillary flow resumed after the neutrophils passed through the capillaries. In contrast, in the sepsis-induced ALI model of mice in which neutrophil recruitment was augmented, the flow of the cells in the pulmonary microcirculation was interrupted in numerous spots (figure $2 \mathrm{~b}, \mathrm{c}$ and supplementary video S4). Thus, during early inflammation, when innate immune cells are recruited, we identified neutrophils as the primary obstacle in the microcirculation in pulmonary capillaries.

Intravascular neutrophil motility initially increases and later decreases to arrest during the early development of sepsis-induced ALI

To investigate the dynamic behaviours of individual neutrophils during entrapment in the pulmonary capillary, we performed a total of $30 \mathrm{~min}$ of time-lapse imaging of neutrophils in the pulmonary
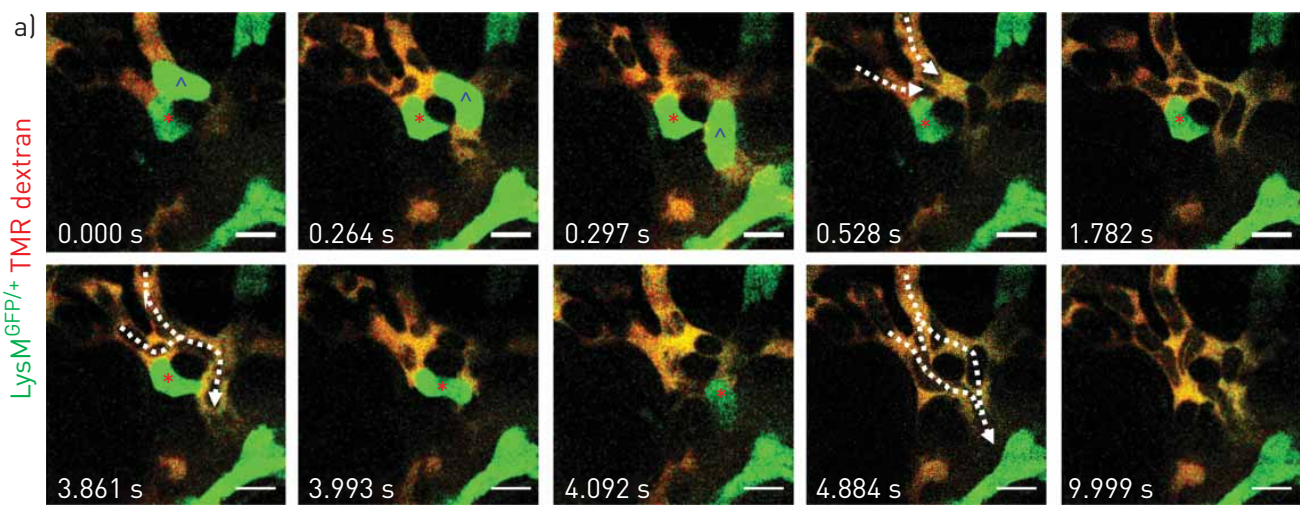

b)

Wide field
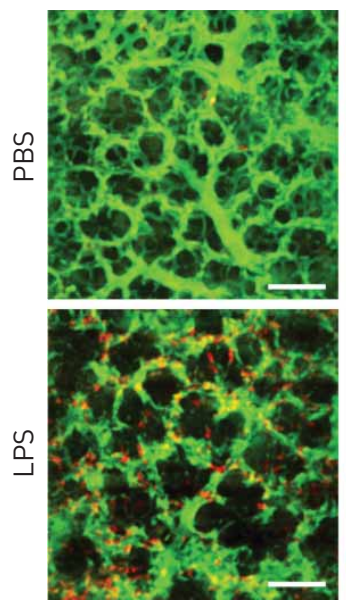

Averaged imaging
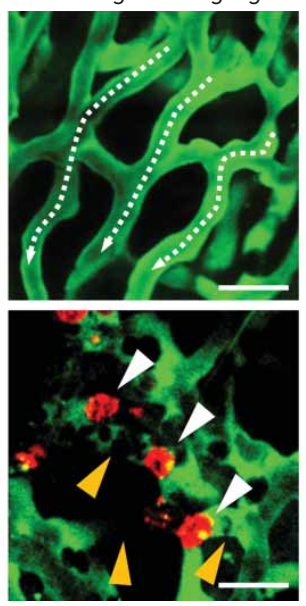

Single frame

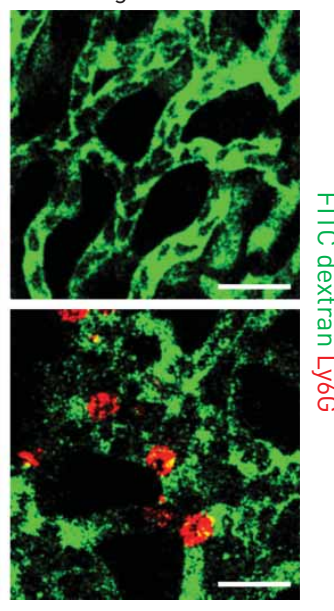

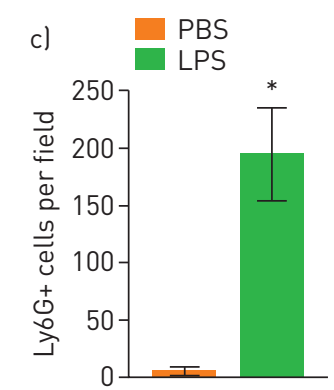

FIGURE 2 Entrapment of neutrophil inside the pulmonary capillary. a) Real-time imaging of LysM GFP/+ (green) neutrophil entrapment in the pulmonary capillary (tetramethylrhodamine (TMR) dextran, red) (supplementary video S3). Each circulation inside the capillary resumes after the neutrophil in the upper region (blue caret) and the lower region (red asterisk) has squeezed through the pulmonary capillary. Scale bars, $10 \mu \mathrm{m}$. Dashed arrows indicate the direction of flow. Time elapsed is indicated. b) Representative intravital imaging of Ly6G+ cells (red) and consequent flow in pulmonary microcirculation (fluorescein isothiocyanate (FITC) dextran, green) in the PBS and lipopolysaccharide (LPS) $\left(10 \mathrm{mg} \cdot \mathrm{kg}^{-1}\right.$ ) groups (supplementary video S4). Magnified spots consist of averaged imaging up to 30 frames and single frame imaging. Dashed arrows indicate the direction of flow. White arrowheads indicate entrapped neutrophils, and yellow arrowheads indicate obstructed capillary with no flow. Scale bars, $100 \mu \mathrm{m}$ (wide field) and $20 \mu \mathrm{m}$ (magnified spot). c) Comparison of number of Ly6G+ cells in pulmonary microcirculation between the PBS and LPS groups $(n=30,10$ fields of view per mouse, three mice per group, two-tailed t-test, $\left.{ }^{*} p<0.05\right)$. Data are presented as mean \pm SD. 
microcirculation $3 \mathrm{~h}$ and $6 \mathrm{~h}$ after LPS administration (figure 3a, b and supplementary video S5). Given that the flow velocity of the erythrocytes was high $\left(>500 \mu \mathrm{m} \cdot \mathrm{s}^{-1}\right)$, we determined that neutrophils that were detected continuously for more than 2 min were not flowing; rather, they were sequestered (i.e. crawling or marginating inside the vessel). Time-lapse imaging showed that the proportion of sequestered neutrophils, as well as their individual sequestration times in the LPS group, dramatically increased compared to the PBS control group, in which most of the neutrophils were sequestered briefly (figure $3 \mathrm{c}, \mathrm{d}$ ). Interestingly, during sequestration, the motility of the $3 \mathrm{~h}$ LPS neutrophils increased, as determined by the track displacement length (figure $3 \mathrm{~b}$, e), track length (figure $3 \mathrm{f}$ ) and mean velocity (figure $3 \mathrm{~g}$ ). However, as sepsis progressed, the $6 \mathrm{~h}$ LPS neutrophils became less motile, and the track length and velocity decreased. In addition, the meandering index decreased in a timely manner, which was influenced by an increased sequestration time and the arrest characteristics of the neutrophils (figure $3 \mathrm{~h}$ ). Taken together, these data show that, during the early period of endotoxin-induced ALI, neutrophils begin to activate and become motile inside the capillaries; however, in the late period, they gradually begin to arrest inside the capillaries.

$$
\text { a) }
$$

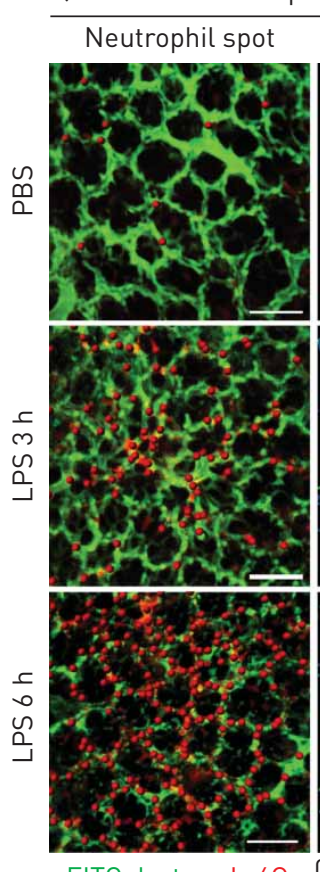

FITC dextran Ly6G

Track
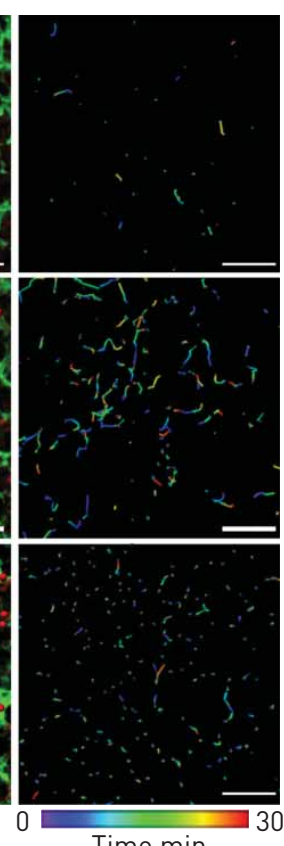

Time min b)

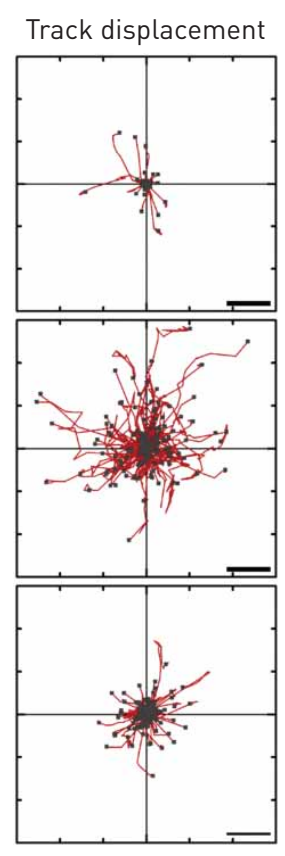

c)

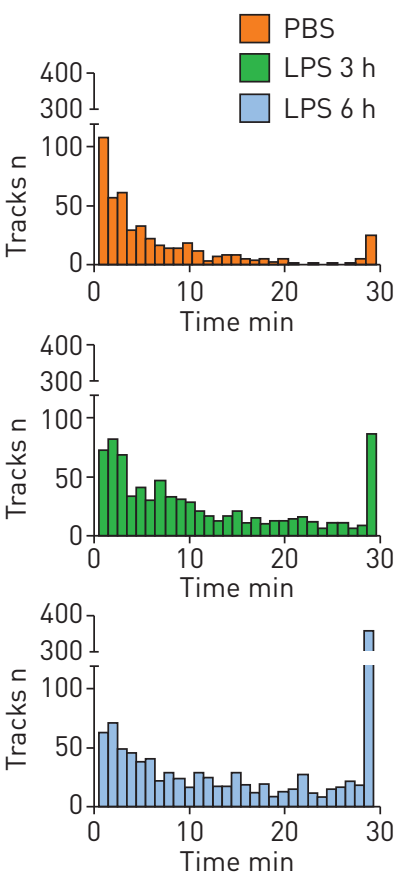

a

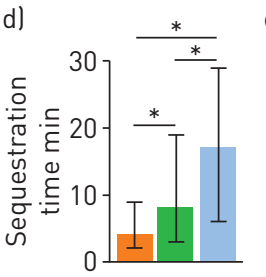

PBS
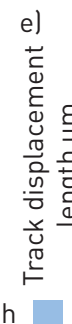

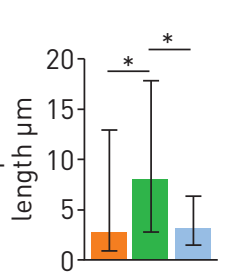

LPS $6 \mathrm{~h}$ f)

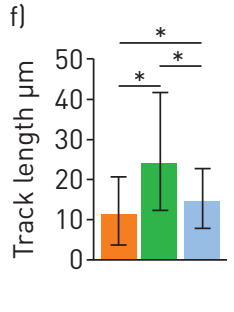

g)

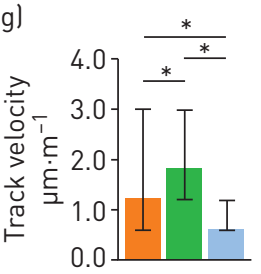

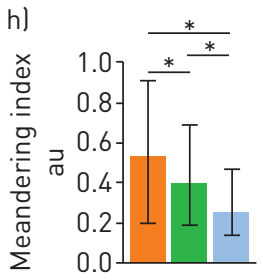

FIGURE 3 Increased entrapment time and dynamically altered motility of neutrophils during the development of sepsis-induced acute lung injury. a) Representative time-lapse imaging of Ly6G+ cells (red spots) in the pulmonary microcirculation (fluorescein isothiocyanate (FITC) dextran, green) in the PBS and lipopolysaccharide (LPS) $3 \mathrm{~h}$ and $6 \mathrm{~h}$ groups (supplementary video S5). The colour-coded track describes the motion of tracked Ly6G+ cells over a period of $30 \mathrm{~min}$. Scale bars, $100 \mu \mathrm{m}$. b) Overlay of the track of Ly6G+ cells from (a). Each Ly6G+ cell track in (a) is plotted from the central point and shows XY displacement. Scale bars, $10 \mu \mathrm{m}$. c) Histogram of track duration of Ly6G+ cells shown in (a). d-h) Comparisons of sequestration time, track displacement length, track length, track velocity and meandering index of Ly6G+ cells in the pulmonary microcirculation in the PBS $(n=466)$ and LPS $3 \mathrm{~h}(\mathrm{n}=794)$ and $6 \mathrm{~h}(\mathrm{n}=1076)$ groups (three mice per group, Kruskal-Wallis test with post hoc Dunn's multiple comparison test, $\left.{ }^{*} p<0.05\right)$. Data are presented as median (interquartile range). 
Neutrophils obstruct pulmonary vessels, generate circulatory dead space and release ROS in situ in sepsis-induced ALI

Using the intravital imaging of the pulmonary microcirculation 3-6 h after LPS administration, we observed for the first time the entire process of dead space formation in the pulmonary microcirculation. In the capillaries, a circulating neutrophil became trapped on one side of a vessel in which the other side was already obstructed by another neutrophil, as revealed by real-time imaging (figure $4 \mathrm{a}$ and supplementary video S6). The flow stopped between the two neutrophils, thereby generating a circulatory dead space. At some capillary sites, we observed clusters of neutrophils for which no flow was detected (supplementary figure S2a). Such obstructions were not limited to the capillaries, but were also observed in the branching regions of the arterioles that connected to the capillaries (supplementary figure S2b, c). Over the course of $10 \mathrm{~min}$ of time-lapse intravital imaging, we observed that neutrophils quickly blocked the branching sites and disturbed the microcirculation near the obstructed region (figure $4 \mathrm{~b}$ and supplementary video S7). To confirm the correlation of neutrophil sequestration and dead space formation, we injected DiD-labelled erythrocytes and visualised the functional capillary (figure $4 \mathrm{c}$ and supplementary video S8). Functional capillary mapping and track path analysis demonstrated that neutrophil-induced capillary and arteriole obstruction generated circulatory dead space.

To identify the function and activation of the entrapped neutrophils, we investigated ROS production in the sequestered neutrophils. Using our intravital imaging system, we detected ROS generation in intravascular neutrophils in the lungs in situ (figure 4d) [26]. Compared to the control group, in which ROS was undetectable in transiently sequestered neutrophils, both the number and proportion of ROS-generating neutrophils were dramatically increased in the LPS-treated mice (figure $4 \mathrm{e}, \mathrm{f}$ ). In contrast to the previous understanding of ROS production by neutrophils at the site of inflammation [27], our findings suggest that ROS production is initiated at a much earlier stage because of the development of neutrophil entrapment in the capillary. The findings also imply that entrapped neutrophils could release ROS in situ, which could harm the endothelial cells and adjacent intravascular structure before extravasation.

Neutrophil depletion improves the pulmonary microcirculation in sepsis-induced ALI

To further confirm the essential role of neutrophils in capillary blockade, we investigated the pulmonary microcirculation in a neutrophil-depleted (N-dep) mouse model. Injecting $200 \mu \mathrm{g}$ of a Ly6G-specific monoclonal antibody (1A8) $24 \mathrm{~h}$ before imaging led to neutrophil depletion (supplementary figure S3) [28]. Using antibodies, neutrophils were depleted in the $\mathrm{LysM}^{\mathrm{GFP} /+}$ mice $24 \mathrm{~h}$ before LPS injection, and then intravital imaging was performed $6 \mathrm{~h}$ after LPS injection (figure 5a). Consistent with our hypothesis, neutrophil depletion improved the FCR in the LPS treatment group; this was repeatedly verified by magnified imaging (figure 5b, c and supplementary video S9). The number of LysM+ cells decreased to a certain level after neutrophil depletion, but there was not complete depletion because of remnant LysM+ cells (figure $5 \mathrm{~d}$ ), which were presumably alveolar macrophages in the extravascular spaces (figure $5 \mathrm{~b}$, magnified) $[24,25]$. Nevertheless, our results indicate that the decreased number of LysM+ cells, mostly intravascular neutrophils, leads to improved FCRs in the pulmonary microcirculation (figure $5 \mathrm{~d}$ ). These data support the idea that neutrophils function as the main components of aggregates and the primary blockers of flow in the pulmonary microcirculation during systemic inflammation.

Mac-1-upregulated neutrophils are sequestered in the lung in sepsis-induced ALI

Although neutrophil depletion increased the FCR, bench-to-bedside clinical translation of the neutrophil depletion strategy is not feasible [13]. Therefore, to identify a target for subsets of sequestered neutrophils, we isolated two groups of neutrophils in a single mouse (figure 6a). We hypothesised that neutrophils in the left ventricle, which have already passed through the pulmonary capillary, would have a different integrin expression pattern from neutrophils in the lung, where sequestration occurs. Flow cytometry was performed for the two groups of neutrophils gated on Ly6G+ to investigate their integrin expression (figure $6 \mathrm{~b}$ and supplementary figure S4). We found that the baseline expression of CD11b and CD18 was upregulated in the neutrophils in the LPS group. Interestingly, in the same mouse, the neutrophils in the lung expressed higher levels of CD11b and CD18 than the neutrophils in the left ventricle (figure $6 c-f$ ). Intravital pulmonary imaging (figure $6 \mathrm{~g}, \mathrm{~h}$ ) confirmed that neutrophils sequestered in the lung in LPS mice expressed high levels of CD11b and CD18, in agreement with our flow cytometry findings (figure 6i-1). Thus, compared to circulating neutrophils, neutrophils sequestered in the lung had upregulated expression of Mac-1 (CD11b/CD18) integrins in the sepsis-induced ALI model.

A Mac-1 inhibitor restores the FCR in the pulmonary microcirculation in sepsis-induced ALI Given that Mac-1 is upregulated in neutrophils that are sequestered in the lung, we investigated the effects of a Mac-1 inhibitor on the pulmonary microcirculation. To extend our findings to a polymicrobial sepsis 
a)

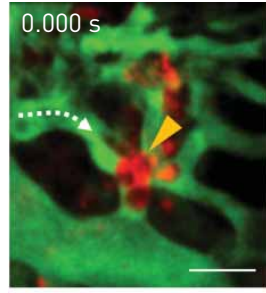

b)

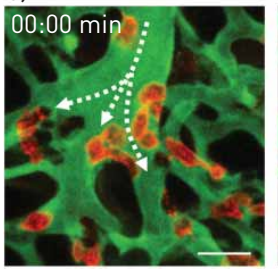

c)

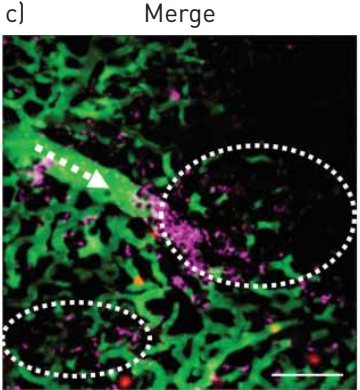

FITC dextran Ly6G DiD-RBC

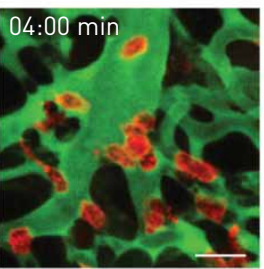

Track path

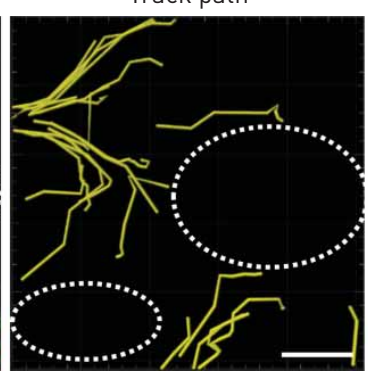

d) Neutrophil
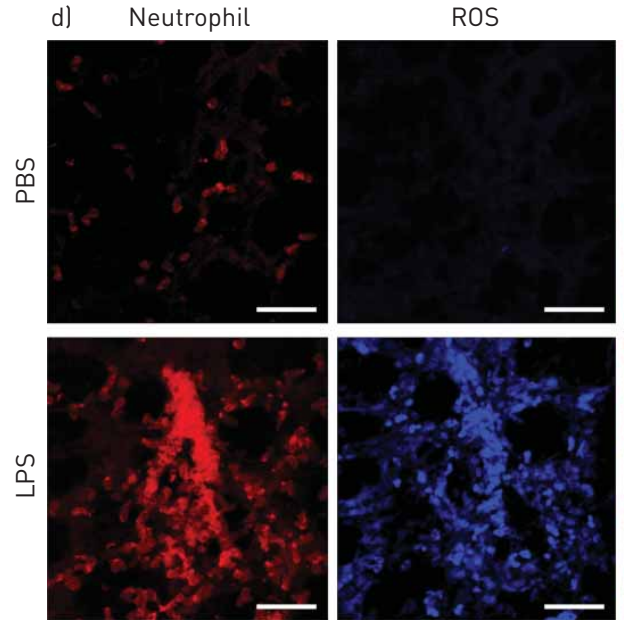

Ly6G

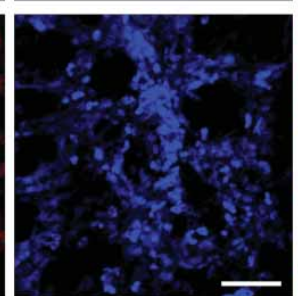

DHE
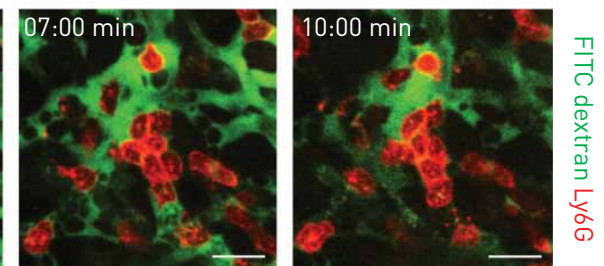

Functional capillary

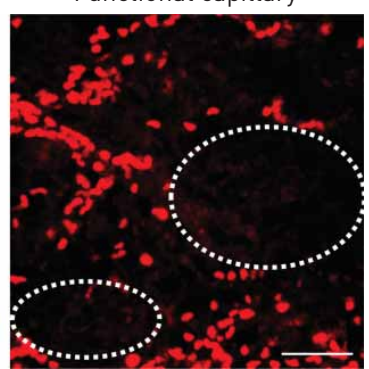

DiD-RBC

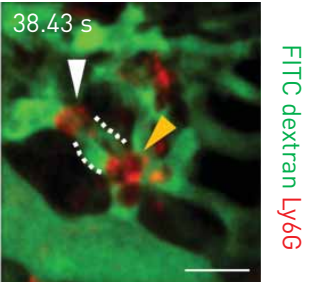


a)

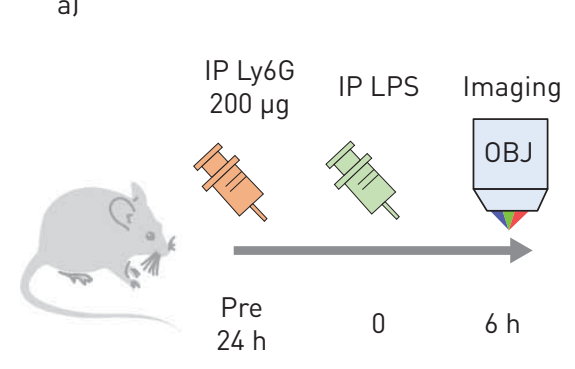

c)

PBS

LPS

N-dep

N-dep+LPS

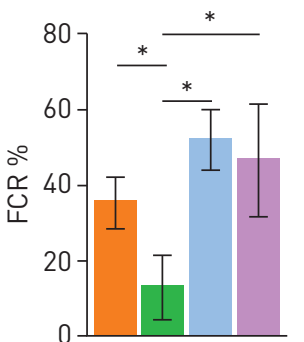

d)

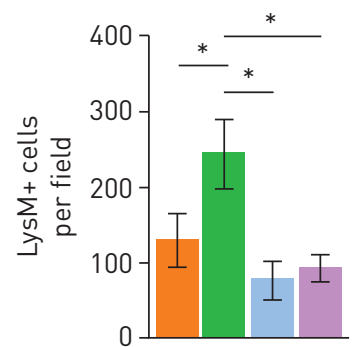

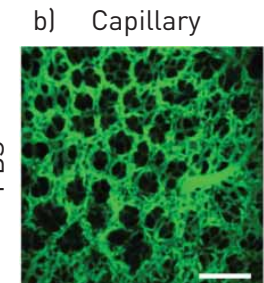

Functional
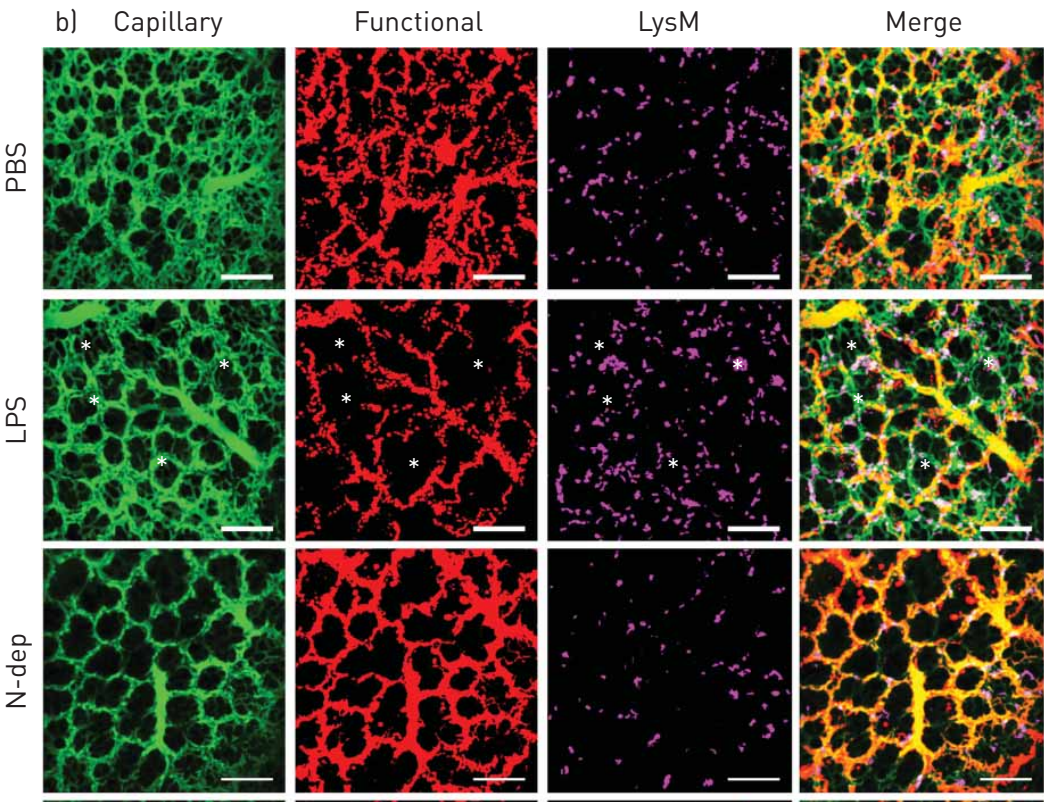

Magnified
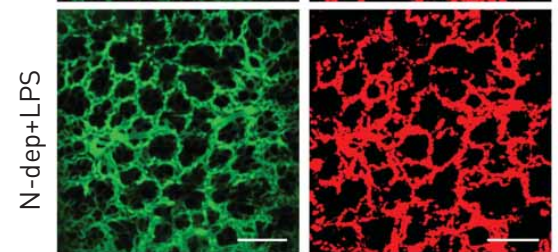

TMR dextran

DiD-RBC

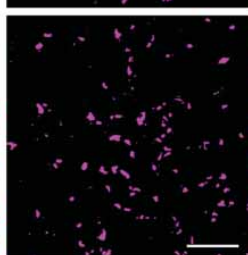

LysM

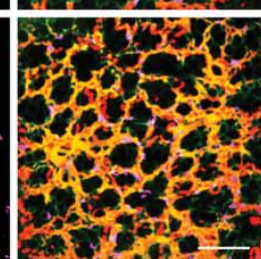

TMR dextran LysM DiD-RBC
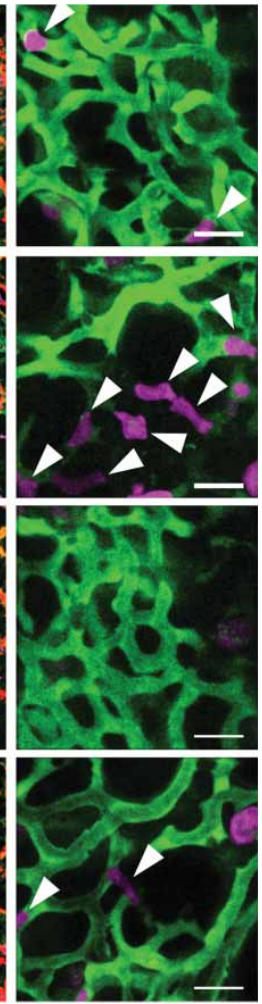

TMR dextran LysM

FIGURE 5 Neutrophil depletion ( $N$-dep) improves the functional capillary ratio (FCR) of the pulmonary microcirculation in a sepsis-induced acute lung injury (ALI) model. a) Schematics of intravital lung imaging of $\mathrm{N}$-dep in a sepsis-induced ALI model. b) Representative intravital imaging of FCR in the pulmonary microcirculation in the PBS, lipopolysaccharide (LPS), N-dep and N-dep+LPS group. Anatomical capillary (tetramethylrhodamine (TMR) dextran, green), functional capillary (DiD-labelled erythrocyte, red) and LysM+ (LysM $\mathrm{GFP} /+^{\text {, }}$ magenta) cell imaging was acquired. White asterisks indicate dead spaces. Magnified images show entrapped LysM+ cells (arrowheads) and consequent flow disturbance (supplementary video S9). Scale bars, $20 \mu \mathrm{m}$ in magnified, otherwise, $100 \mu \mathrm{m}$. c, d) Comparisons of FCR (\%) and LysM+ cell count among PBS, LPS, N-dep and N-dep+LPS groups (n=30, 10 fields of view per mouse, three mice per group, one-way ANOVA with post hoc Holm-Sidak's multiple comparisons test, $\left.{ }^{*} p<0.05\right)$. Data are presented as mean \pm SD. IP: intraperitoneal; OBJ: objective lens; RBC: red blood cells.

model, we performed intravital lung imaging on a CLP mouse model. As in the LPS model, the FCR was significantly lower in the CLP mouse model than in the control (figure 7a, b). Administering an anti-CD11b antibody (5 mg. $\mathrm{kg}^{-1}$, intraperitoneal) [29] and abciximab (10 mg. $\mathrm{kg}^{-1}$, intraperitoneal) [30-32], a cross-reactive inhibitor for the binding of various ligands to Mac-1, restored the FCR with less sequestration of Ly6G+ cells (figure 7c). To further confirm our findings, the FCR was calculated at the same site and was significantly increased after the injection of abciximab (10 $\mathrm{mg} \cdot \mathrm{kg}^{-1}$, intravenous) 
a)
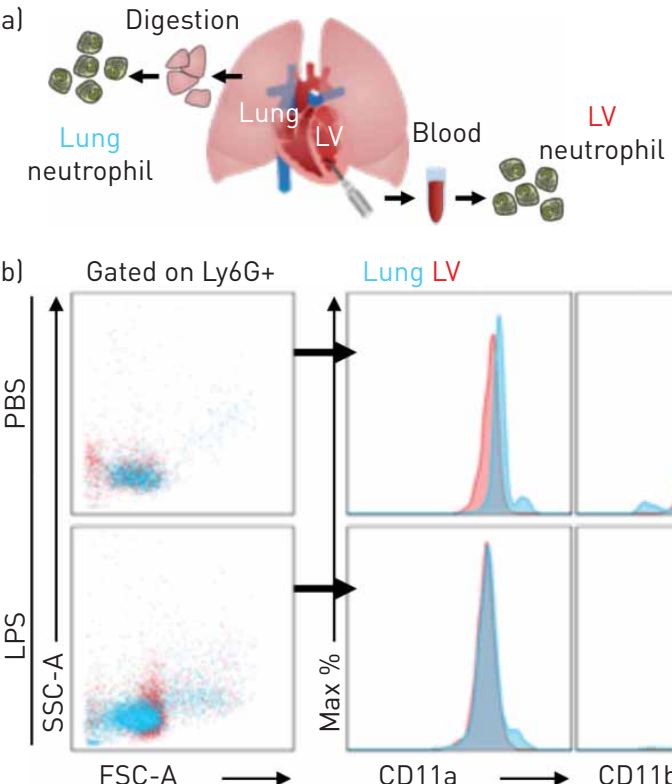

Lung LV
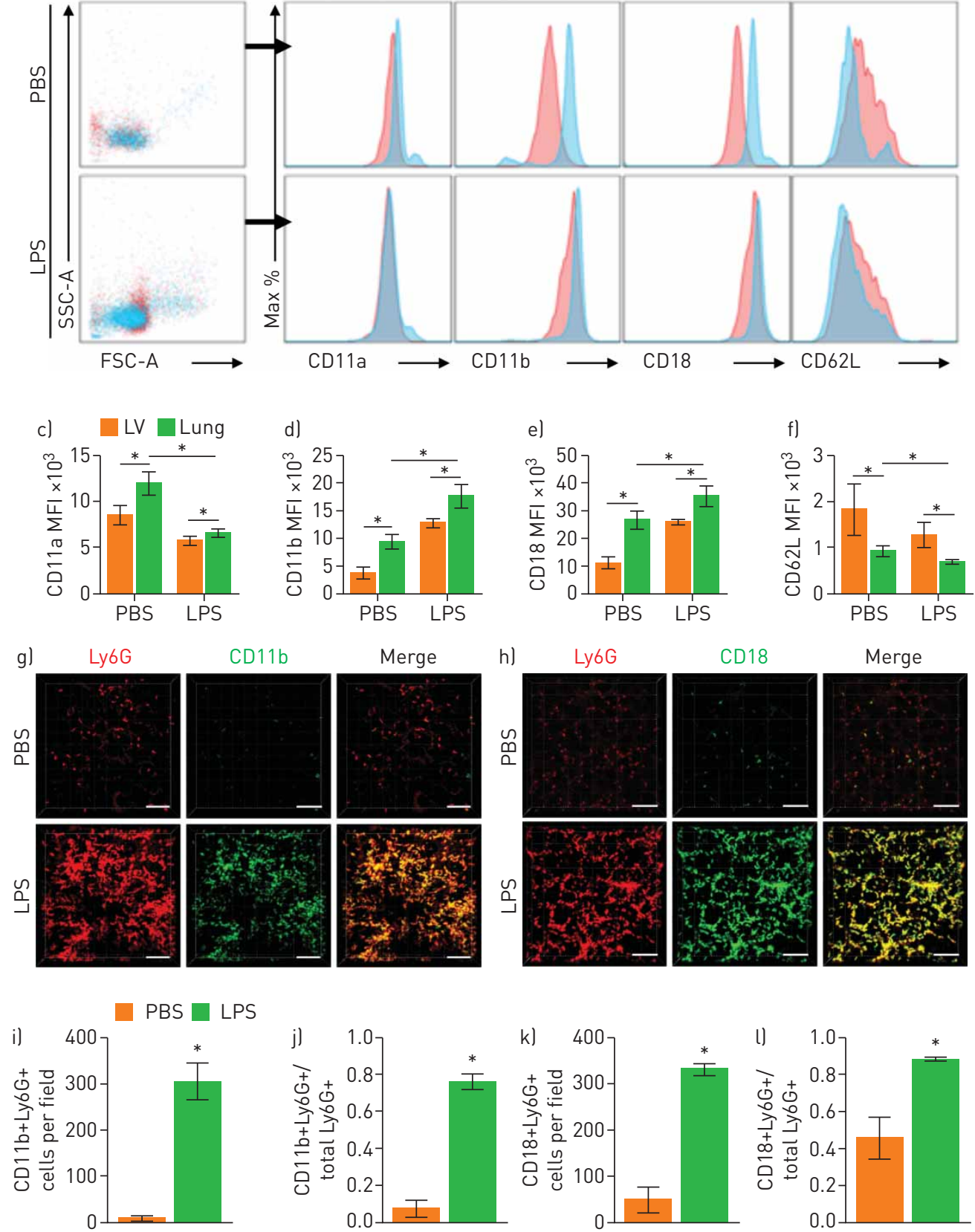

FIGURE 6 Mac-1 integrin is upregulated in entrapped neutrophils in the pulmonary microcirculation. a) Schematic diagram showing isolation sampling of two groups of neutrophils (lung, blue; left ventricle (LV), red). b) Surface expression of integrin of Ly6G+ cells from the lung and LV. C-f) Comparisons of expression of integrin from flow cytometry in the PBS and lipopolysaccharide (LPS) groups ( $n=5$ per each group, MannWhitney test, ${ }^{*} p<0.05$ ). Data are presented as mean \pm SD. $g$, h) Representative three-dimensional intravital imaging of integrin in sequestered neutrophils. Cellular surface expression of CD11b (green) and CD18 (green) in Ly6G+ cells (red) is visualised in vivo. Scale bars, $100 \mu \mathrm{m}$. $\mathrm{i}-\mathrm{l}$ ) Comparisons of number of CD11b +Ly6G+ cells and CD18+Ly6G+ cells and ratios of CD11b+Ly6G+ cells and CD18+Ly6G+ cells over total Ly6G+ cells in the PBS and LPS groups ( $n=9$, three fields of view per mouse, three mice per group, Mann-Whitney test, $\left.{ }^{*} p<0.05\right)$. Data are presented as mean $\pm S D$. SSC-A: side scatter-area; MFI: mean fluorescence intensity. 
a)
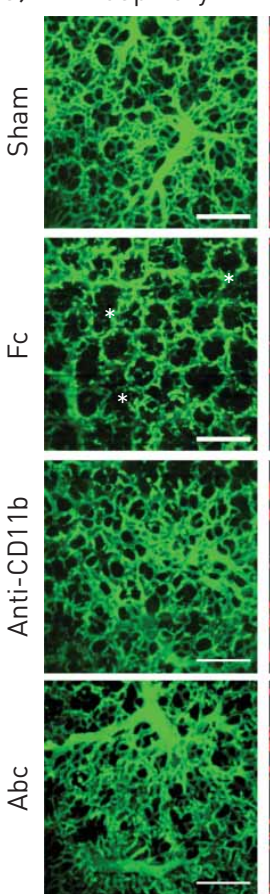

FITC dextran
Functional
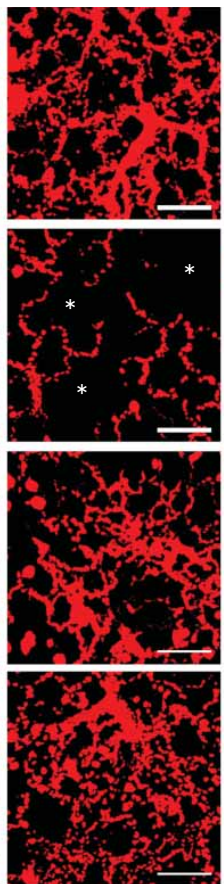

DiD-RBC
Ly6G
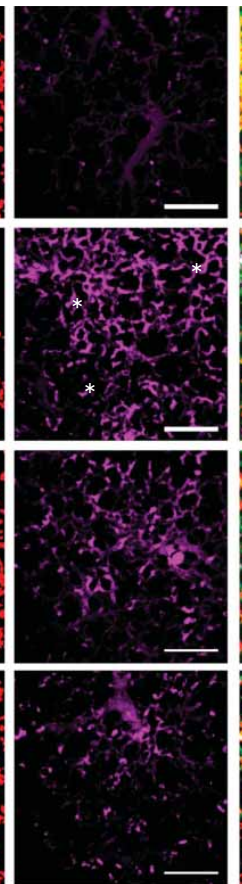

Ly6G
Merge
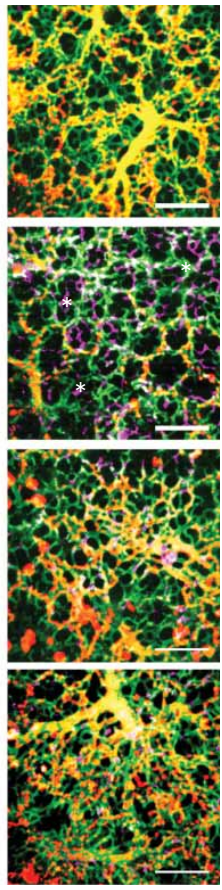

FITC dextran

Ly6G DiD-RBC b)
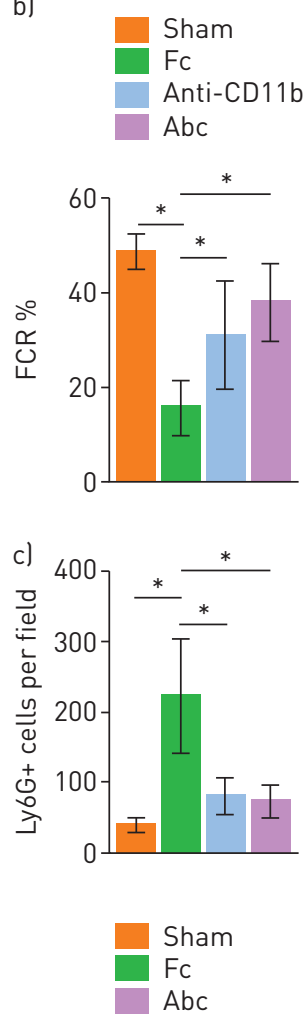

d)

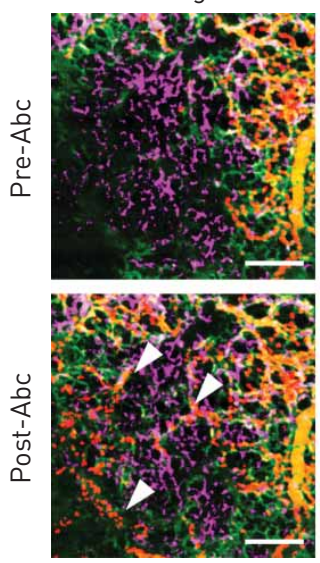

FITC dextran

Ly6G DiD-RBC
Functional
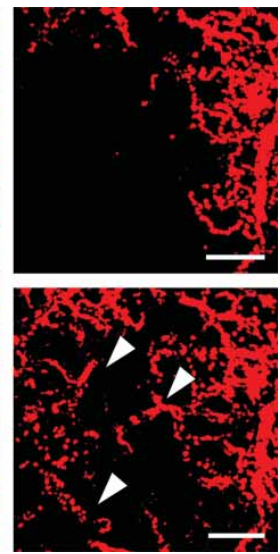

DiD-RBC e)
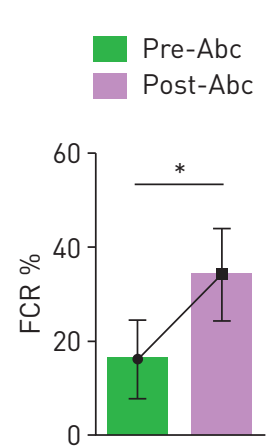
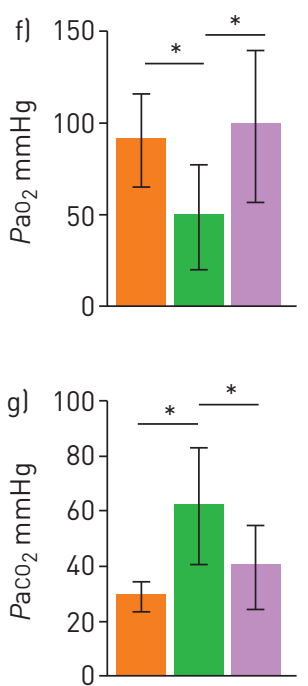

FIGURE 7 Mac-1 inhibitor ameliorates the functional capillary ratio (FCR) of the pulmonary microcirculation in sepsis-induced acute lung injury. a) Representative intravital imaging of FCR in the pulmonary microcirculation in the sham, fragment crystallisable (Fc), Anti-CD11b and abciximab (Abc) groups. Anatomical capillary (tetramethylrhodamine (TMR) dextran, green), functional capillary (DiD-labelled erythrocytes, red) and neutrophil (Ly6G, magenta) imaging was acquired. White asterisks indicate dead spaces. Scale bars, $100 \mu \mathrm{m}$. b, c) Comparisons of FCR and number of Ly6G+ cells in the pulmonary microcirculation ( $n=14-25$, three mice per group, two-tailed t-test, $\left.{ }^{*} p<0.05\right)$. Data are presented as mean \pm SD. d) Representative intravital lung imaging of the pre- and post-Abc groups (supplementary video S10). White arrowheads indicate restoration of erythrocyte perfusion. Scale bars, $100 \mu \mathrm{m}$. el Comparison of FCR in the pre- and post-Abc groups $(n=20$ and $24,6-8$ fields of view per mouse, three mice per group, two-tailed t-test, $\left.{ }^{*} \mathrm{p}<0.05\right)$. Data are presented as mean \pm SD. $\left.\mathrm{f}, \mathrm{g}\right)$ Comparisons of the arterial oxygen $\left(\mathrm{PaO}_{2}\right)$ and carbon dioxide $\left(\mathrm{PaCO}_{2}\right)$ tension in the sham $(n=8), \mathrm{Fc}(\mathrm{n}=10)$ and $\mathrm{Abc}(\mathrm{n}=6)$ groups (Kruskal-Wallis test with post hoc Dunn's multiple comparison tests, $\left.{ }^{*} p<0.05\right)$. Data are presented as mean \pm SD. RBC: red blood cells.

(figure 7d, e and supplementary video S10). Arterial blood gas analysis in the left ventricle confirmed that the FCR results were in accordance with hypoxaemia and hypercapnia, suggesting that abciximab increases gas exchange in this sepsis-induced ALI mouse model through an improvement in the microcirculation 
(figure $7 \mathrm{f}, \mathrm{g}$ ). Furthermore, pulmonary oedema after $24 \mathrm{~h}$ of injury was significantly ameliorated in the Mac-1 inhibitor model, in accordance with previous results (supplementary figure S5).

\section{Discussion}

Despite differences between the systemic and pulmonary capillary network, most of our knowledge of the microcirculation in sepsis has come from studies of the systemic circulation, with decreased functional capillary density and increased intercapillary areas [33]. Although our study of the pulmonary microcirculation during sepsis partially agrees with findings from the systemic circulation, the underlying aetiology of the microcirculatory change is distinct from that of the systemic circulation. Endothelial dysfunction and vasoconstriction have previously been suggested as potential central mechanisms in impaired systemic microcirculation [34]. Our results suggest that cluster formation by recruited neutrophils also has a key detrimental role in the pulmonary microcirculation in the early stage of sepsis-induced ALI.

Even though previous studies have suggested that sequestration in pulmonary capillaries functions as a defensive immune surveillance system to detect pathogens in the circulation, this does not explain how neutrophil sequestration progresses to ARDS $[14,35]$. By contrast, our study shows that neutrophils form clusters and obstruct capillaries and arterioles, leading to the redistribution and obstruction of the pulmonary microcirculation, highlighting the novel detrimental role of neutrophils. Recently, studies have revealed clusters of neutrophils inside the pulmonary vasculature in lung biopsies from patients with ARDS [10] and murine fungal sepsis [36], which supports our results regarding the aggregate formation of neutrophils. The neutrophil-induced obstruction of flow increases the mismatching areas of ventilation and perfusion, thereby intensifying hypoxia due to sepsis-induced ARDS. Compared to previous intravital imaging studies on the adhesion of neutrophils in the pulmonary capillary $[37,38]$, our study clearly shows how dead space with ventilation-perfusion mismatch is created in the microcirculation by neutrophils. Moreover, we can directly image the dead space fraction, which has been estimated indirectly by the difference in the partial pressure of arterial versus exhaled carbon dioxide using volumetric capnography.

Although our results imply that neutrophil depletion improves the pulmonary circulation, there is considerable debate about the effects of treatment-induced neutrophil depletion in sepsis because the effects on bacterial clearance and the response to systemic inflammation are unclear [39]. Accordingly, we evaluated the subpopulation of neutrophils that might ameliorate lung injury [40]. Flow cytometry showed that Mac-1 (CD11b/CD18), which interacts with ICAM-1 in endothelial cells and various coagulation factors, was significantly upregulated in the sequestered neutrophils in lungs from the ALI model. Mac-1 could, therefore, be a potential target integrin for improving the pulmonary microcirculation. Previous studies have investigated the role of L-selectin and CD11/CD18 in neutrophil sequestration and revealed that neither was required for the initial immediate sequestration occurring within $1 \mathrm{~min}$ in the pulmonary capillaries. However, once the neutrophils were sequestered, L-selectin and CD11/CD18 were critical for the adherence of neutrophils within the capillary bed for more than 4-7 min [14, 41]. Additionally, several studies have suggested the inhibition of Mac-1 as a treatment for protection against neutrophil infiltration in lung injury during sepsis $[42,43]$. In contrast to indirect assays utilised in previous studies, our research used real-time intravital microscopic imaging to show that the inhibition of Mac-1 reduced neutrophil sequestration, increased the FCR and offered protection from ALI. However, a recent study reported on neutrophil-platelet interactions in thrombosis in the pulmonary vasculature $[10,44]$, raising concerns about whether platelets in thrombosis may have a confounding effect and whether the effects of abciximab, also known as glycoprotein IIb/IIIa inhibitor, are the consequence of thrombus resolution due to interactions with platelets. There may be platelets that were not identified in our study; nevertheless, most of the flow disturbances we observed were resolved by neutrophil depletion. This implies that neutrophils have an essential role in augmenting cluster formation inside pulmonary capillaries in sepsis-induced ALI [36]. Moreover, because we found that anti-CD11b and abciximab elicited equivalent improvements in the FCR, it seems that the effects of abciximab could be attributed to its anti-Mac-1 activity on neutrophils. Nevertheless, owing to the unavailability of platelet imaging, we were not able to identify the involvement of platelets in the formation of neutrophil cluster bridging between neutrophils. We could not, therefore, rule out the additional effect of abciximab targeting not only the neutrophil through $\mathrm{CD} 11 \mathrm{~b}$ but also the platelets through glycoprotein IIb/IIIa. Additional intravital lung imaging should be performed to further elucidate neutrophil-platelet thrombogenesis in vivo to gain a better understanding of the relevant pathophysiology and subsequent molecular targets in the pulmonary vasculature. Furthermore, abciximab has the advantage that is has already been approved by the US Food and Drug Administration for use during coronary intervention to prevent thrombosis, which brings our study much closer to the clinical field. 
In conclusion, the use of a custom-made real-time intravital lung microscopic imaging system enabled direct visualisation of prolonged neutrophil entrapment in capillaries during sepsis-induced ALI in mice. The resulting disturbance in flow in the pulmonary microcirculation correlated with dead space. This finding provides novel insights into how capillary entrapment of neutrophils contributes to pulmonary microcirculatory disturbances. This system could serve as a useful tool for investigating diseases that affect the pulmonary microcirculation and could be used to evaluate potential treatments for sepsis-induced ARDS.

Acknowledgements: The authors thank Seonghye Kim (SNUBH) for her technical assistance during revisional experiments. The authors also thank Soyeon Ahn, Jieun Moon, Eunji Kong, Jingu Lee, Ryul Kim and Sujung Hong (KAIST) for advice and helpful discussion; and Soo Yun Lee and Haeun Kim (KAIST) for their technical assistance.

Conflict of interest: None declared.

Support statement: This research was supported by the Health Fellowship Foundation and the Global PhD Fellowship Program (NRF-2015H1A2A1030717) through the National Research Foundation of Korea (NRF) funded by the Ministry of Education, Republic of Korea; the Global Frontier Project (NRF-2013M3A6A4044716), Basic Research Program (NRF-2017R1E1A1A01074190) funded by the Ministry of Science and ICT, Republic of Korea; and Korea Healthcare Technology R\&D Project (HI15C0399) funded by the Ministry of Health and Welfare, Republic of Korea. Funding information for this article has been deposited with the Crossref Funder Registry.

\section{References}

1 Torio CM, Moore BJ. National inpatient hospital costs: the most expensive conditions by payer, 2013: statistical brief \#204. In:Healthcare Cost and Utilization Project (HCUP) Statistical Briefs. Rockville, MD, Agency for Healthcare Research and Quality (US), 2016.

2 Hall MJ, Levant S, DeFrances CJ. Trends in inpatient hospital deaths: National Hospital Discharge Survey, 20002010. NCHS Data Brief 2013; 118:1-8.

3 Singer M, Deutschman CS, Seymour CW, et al. The third international consensus definitions for sepsis and septic shock (Sepsis-3). JAMA 2016; 315: 801-810.

4 Angus DC, van der Poll T. Severe sepsis and septic shock. N Engl J Med 2013; 369: 840-851.

5 Lagu T, Rothberg MB, Shieh MS, et al. Hospitalizations, costs, and outcomes of severe sepsis in the United States 2003 to 2007. Crit Care Med 2012; 40: 754-761.

6 Thompson BT, Chambers RC, Liu KD. Acute respiratory distress syndrome. N Engl J Med 2017; 377: 562-572.

7 Nuckton TJ, Alonso JA, Kallet RH, et al. Pulmonary dead-space fraction as a risk factor for death in the acute respiratory distress syndrome. N Engl J Med 2002; 346: 1281-1286.

8 Ryan D, Frohlich S, McLoughlin P. Pulmonary vascular dysfunction in ARDS. Ann Intensive Care 2014; 4: 28.

9 Matthay MA, Ware LB, Zimmerman GA. The acute respiratory distress syndrome. J Clin Invest 2012; 122: 2731-2740.

10 Yuan Y, Alwis I, Wu MCL, et al. Neutrophil macroaggregates promote widespread pulmonary thrombosis after gut ischemia. Sci Transl Med 2017; 9: pii: eaam5861.

11 Grommes J, Soehnlein O. Contribution of neutrophils to acute lung injury. Mol Med 2011; 17: 293-307.

12 Matute-Bello G, Downey G, Moore BB, et al. Acute Lung Injury in Animals Study Group. An official American Thoracic Society workshop report: features and measurements of experimental acute lung injury in animals. Am J Respir Cell Mol Biol 2011; 44: 725-738.

13 Phillipson M, Kubes P. The neutrophil in vascular inflammation. Nat Med 2011; 17: 1381-1390.

14 Doerschuk CM. Mechanisms of leukocyte sequestration in inflamed lungs. Microcirculation 2001; 8: 71-88.

15 MacNee W, Selby C. New perspectives on basic mechanisms in lung disease. 2. Neutrophil traffic in the lungs: role of haemodynamics, cell adhesion, and deformability. Thorax 1993; 48: 79-88.

16 Kuebler WM, Borges J, Sckell A, et al. Role of L-selectin in leukocyte sequestration in lung capillaries in a rabbit model of endotoxemia. Am J Respir Crit Care Med 2000; 161: 36-43.

17 Lien DC, Henson PM, Capen RL, et al. Neutrophil kinetics in the pulmonary microcirculation during acute inflammation. Lab Invest 1991; 65: 145-159.

18 Looney MR, Bhattacharya J. Live imaging of the lung. Annu Rev Physiol 2014; 76: 431-445.

19 Kim P, Puoris'haag M, Cote D, et al. In vivo confocal and multiphoton microendoscopy. J Biomed Opt 2008; 13: 010501.

20 Han S, Lee SJ, Kim KE, et al. Amelioration of sepsis by TIE2 activation-induced vascular protection. Sci Transl Med 2016; 8: 335ra355.

21 Veilleux I, Spencer JA, Biss DP, et al. In vivo cell tracking with video rate multimodality laser scanning microscopy. IEEE J Select Top Quant Electron 2008; 14: 10-18.

22 Park I, Choe K, Seo H, et al. Intravital imaging of a pulmonary endothelial surface layer in a murine sepsis model. Biomed Opt Express 2018; 9: 2383-2393.

23 Seo $\mathrm{H}$, Hwang $\mathrm{Y}$, Choe $\mathrm{K}$, et al. In vivo quantitation of injected circulating tumor cells from great saphenous vein based on video-rate confocal microscopy. Biomed Opt Express 2015; 6: 2158-2167.

24 Faust N, Varas F, Kelly LM, et al. Insertion of enhanced green fluorescent protein into the lysozyme gene creates mice with green fluorescent granulocytes and macrophages. Blood 2000; 96: 719-726.

25 Kreisel D, Nava RG, Li W, et al. In vivo two-photon imaging reveals monocyte-dependent neutrophil extravasation during pulmonary inflammation. Proc Natl Acad Sci USA 2010; 107: 18073-18078.

26 Finsterbusch M, Hall P, Li A, et al. Patrolling monocytes promote intravascular neutrophil activation and glomerular injury in the acutely inflamed glomerulus. Proc Natl Acad Sci USA 2016; 113: E5172-E5181.

27 Mittal M, Siddiqui MR, Tran K, et al. Reactive oxygen species in inflammation and tissue injury. Antioxid Redox Signal 2014; 20: 1126-1167. 

mice. J Leukoc Biol 2008; 83. 64-70.

29 Ahn GO, Tseng D, Liao CH, et al. Inhibition of Mac-1 (CD11b/CD18) enhances tumor response to radiation by reducing myeloid cell recruitment. Proc Natl Acad Sci USA 2010; 107: 8363-8368.

30 Peter K, Schwarz M, Conradt C, et al. Heparin inhibits ligand binding to the leukocyte integrin Mac-1 (CD11b/ CD18). Circulation 1999; 100: 1533-1539.

31 Schwarz M, Nordt T, Bode C, et al. The GP IIb/IIIa inhibitor abciximab (c7E3) inhibits the binding of various ligands to the leukocyte integrin Mac-1 (CD11b/CD18, alphaMbeta2). Thromb Res 2002; 107: 121-128.

32 Mickelson JK, Ali MN, Kleiman NS, et al. Chimeric 7E3 Fab (ReoPro) decreases detectable CD11b on neutrophils from patients undergoing coronary angioplasty. J Am Coll Cardiol 1999; 33: 97-106.

33 De Backer D, Creteur J, Preiser JC, et al. Microvascular blood flow is altered in patients with sepsis. Am J Respir Crit Care Med 2002; 166: 98-104.

34 De Backer D, Orbegozo Cortes D, Donadello K, et al. Pathophysiology of microcirculatory dysfunction and the pathogenesis of septic shock. Virulence 2014; 5: 73-79.

35 Yipp BG, Kim JH, Lima R, et al. The lung is a host defense niche for immediate neutrophil-mediated vascular protection. Sci Immunol 2017; 2: pii: eaam8929.

36 Lee EKS, Gillrie MR, Li L, et al. Leukotriene B4-mediated neutrophil recruitment causes pulmonary capillaritis during lethal fungal sepsis. Cell Host Microbe 2018; 23: 121-133.

37 Yang N, Liu YY, Pan CS, et al. Pretreatment with andrographolide pills $\left({ }^{\oplus}\right)$ attenuates lipopolysaccharide-induced pulmonary microcirculatory disturbance and acute lung injury in rats. Microcirculation 2014; 21: 703-716.

38 Gill SE, Rohan M, Mehta S. Role of pulmonary microvascular endothelial cell apoptosis in murine sepsis-induced lung injury in vivo. Respir Res 2015; 16: 109.

39 Robertson CM, Perrone EE, McConnell KW, et al. Neutrophil depletion causes a fatal defect in murine pulmonary Staphylococcus aureus clearance. J Surg Res 2008; 150: 278-285.

40 Lewis SM, Khan N, Beale R, et al. Depletion of blood neutrophils from patients with sepsis: treatment for the future? Int Immunopharmacol 2013; 17: 1226-1232.

41 Kubo H, Doyle NA, Graham L, et al. L- and P-selectin and CD11/CD18 in intracapillary neutrophil sequestration in rabbit lungs. Am J Respir Crit Care Med 1999; 159: 267-274.

42 Gao XP, Liu Q, Broman $\mathrm{M}$, et al. Inactivation of CD11b in a mouse transgenic model protects against sepsis-induced lung PMN infiltration and vascular injury. Physiol Genomics 2005; 21: 230-242.

43 Asaduzzaman M, Zhang S, Lavasani S, et al. LFA-1 and MAC-1 mediate pulmonary recruitment of neutrophils and tissue damage in abdominal sepsis. Shock 2008; 30: 254-259.

44 Bennewitz MF, Jimenez MA, Vats R, et al. Lung vaso-occlusion in sickle cell disease mediated by arteriolar neutrophil-platelet microemboli. JCI Insight 2017; 2: e89761. 